\title{
Momordica charantia (Bitter melon) in Combination with Metformin Potentiates Hypoglycemic and Hypolipidemic Effects in Alloxan-induced Diabetic Rats
}

\author{
Monirul Islam ${ }^{1}$, Md. Saiful Islam ${ }^{2}$, Shaheda Zannah ${ }^{2}$, Golam Sadik $^{2}$ \\ and Mamunur Rashid ${ }^{2}$ \\ ${ }^{1}$ Department of Pharmacy, Pabna University of Science and Technology, Pabna, Bangladesh \\ ${ }^{2}$ Department of Pharmacy, University of Rajshahi, Rajshahi-6205, Bangladesh
}

(Received: 1 March, 2018; Accepted: 10 May, 2018; Published: 31 July, 2018)

\begin{abstract}
The present study was designed to investigate the effects of bitter melon (Momordica charantia) in combination with a standard oral hypoglycemic agent metformin on alloxan induced diabetic rats (AIDRs). Both the plant extract and the drug, individually and in combination, were subjected in vivo for two weeks (short term) and four weeks (long term) treatment protocol to determine blood glucose level, lipid profile and liver glycogen level using Swiss Albino rats. In short term treatment protocol, bitter melon extract (BME) at a dose of 75,150 and $300 \mathrm{mg} / \mathrm{kg}$ body weight (bw) were administered in AIDRs by using oral gavages once daily and dose-dependent antihyperglycemic and antidyslipidemic effects were investigated. This short term study revealed that the most effective dose of BME was found as $300 \mathrm{mg} / \mathrm{kg}$ bw among the three doses. In long term treatment protocol, AIDRs in different groups received fixed dose monotherapy of BME $(300 \mathrm{mg} / \mathrm{kg} \mathrm{bw})$ and metformin $(15 \mathrm{mg} / \mathrm{kg} \mathrm{bw})$ and fixed dose BME $(150 \mathrm{mg} / \mathrm{kg}$ bw) and metformin $(7.5 \mathrm{mg} / \mathrm{kg} \mathrm{bw})$ combination therapy. The study showed that combination therapy significantly decreased the blood glucose level from $18.42 \pm 0.95$ to $6.80 \pm 0.39$ $\mathrm{mmol} / \mathrm{l}$ in comparison to the control group after daily treatment for four weeks. In case of antidyslipidemic effect, combination therapy reduced total cholesterol (34.25\%), triglycerides $(11.92 \%)$ and LDL-cholesterol (57.73\%) levels and increased HDL-cholesterol level (55.48\%) in comparison with their respective control groups. Metformin, BME and their combination preserved the liver glycogen level by $35.21 \%, 22.54 \%$ and $49.01 \%$, respectively in comparison to diabetic control group. These changes were significantly better than those of BME and metformin monotherapy. The results suggested that treatment with combination therapy was more effective than mono-therapy for preventing diabetes as bitter melon extract potentiates the effects of metformin on long term alloxan-induced diabetic rats.
\end{abstract}

Key words: Diabetes, bitter melon, metformin, glycemic control and combination therapy.

\section{Introduction}

Diabetes mellitus is one of the most common chronic diseases affecting millions of people worldwide with a large negative impact on the patient's health (DeFronzo et al., 2005). It is a major threat to global public health that is rapidly getting worse and the biggest impact is on adults of working age in developing countries (WHO and IDF, 2004). It was estimated that in 2017 there were 451 million (age 18-99 years) people with diabetes worldwide. These figures are expected to increase to 693 million by 2045 (Cho et al., 2018).

Clinically diabetes mellitus is associated with a number of long term micro vascular (e.g., retinopathy, nephropathy, and neuropathy) as well as macro-vascular (e.g., myocardial infarction, peripheral vascular disease, and stroke) complications that result in significant morbidity and

Correspondence to: Mamunur Rashid; E-mail: mamun69jp@yahoo.com 
mortality (Lamb et al., 2008; Johansen et al., 2005). Oxidative stress has been reported to play an important role in diabetes mellitus right from its genesis to the development of microvascular complications (Ullah et al., 2016). Recent evidence suggests that diabetes is associated with a high risk of cardiovascular disease (CVD) (Carmena, 2005).

Control of diabetes was based entirely on insulin and oral hypoglycemic drug therapy. But due to development of complications by some patients, necessity of finding of some alternative therapy started. Phytotherapy has long been a source of medicinal products and over the years there have been many attempts to use herbal medicines for the treatment of diabetes (Governa et al., 2016). A combination therapy of orthodox medicine and herbal medicine exhibited a better (synergistic) effect than either medicine alone. Therefore, herbal medicine can complement orthodox therapy in type two diabetes mellitus and provides hope for a cure (Chang et al., 2013). In our previous studies we suggested that diets play an important role in the management of hypoglycemia and dyslipidemia (Ali et al., 2013; Rahman et al., 2013). Among these M. charantia showed antidiabetic activity in streptozotocin induced diabetic rats (Sekar et al., 2005). M. charantia is a good example of folk medicine, which is consumed by diabetic patients in India with the belief that it has a useful hypoglycemic potential, is selected for this study (Sastri, 1962). The most important compound having hypoglycemic activity isolated from bitter melon are polypeptide-p and a mixture of two steroid glycosides referred to as charantin (Khanna et al., 2001).

In present study, the antidiabetic and other beneficial effects of bitter melon extract (BME) were assessed by evaluating the comparative hyporglycemic and hypolipidemic activities of this herb with a standard oral hypoglycemic agent metformin. In this study, we also observed the effect of crude extract of $M$. charantia on liver glycogen in alloxan induced diabetic rats. The current research was aimed at providing a strong view on experimental studies carried out on the most effective and commonly used hypoglycemic drug and traditionally used plant based treatment of bitter melon crude extract alone and combinedly.

\section{Materials and Methods}

Preparation of crude extract: The fresh fruits of $M$ charantia (Bitter melon) were purchased from the local market. The fruits were then washed thoroughly with tap water and cut into thin slices. The sliced pieces were dried completely under the mild sun and grinded with an electric grinder into coarse powder and used for cold extraction. After extraction the yield was found to be about $10 \mathrm{ml} / 100 \mathrm{gm}$ bitter melon powder. The authenticity of $M$. charantia was identified by a plant taxonomist from the Department of Botany, University of Rajshahi.

Selection of animal: Swiss Albino male rats weighing about 110-140 gm, age 2 months were acclimatized to the new environmental condition for a period of one week. During the experimental period the rats were kept in a well ventilated animal house at room temperature of $25^{\circ} \mathrm{C}$ and were supplied with standard pellets from International Centre for Diarrhoeal Disease Research, Bangladesh and fresh drinking water. All the rats were kept in cages and maintained with natural 12 hour light and dark cycle.

Experimental induction of diabetes: Swiss Albino male rats except normal control group were allowed to fast for 12 hours. Hyperglycaemia was induced in each fasted rat by administering alloxan monohydrate $[110 \mathrm{mg} / \mathrm{kg}$ body weight $(\mathrm{BW})]$ in normal saline intraperitoneally. $10 \%$ dextrose was there after administered orally to combat the immediate hypoglycaemia that could occur. After 24 hours blood glucose content was measured by using Clever Check glucose test meter (Bioland, Germany) using blood sample collected from the tail vein of the rats. When the condition of diabetes was established animals with blood glucose levels above 11.1 $\mathrm{mmol} / \mathrm{L}$ was selected for the study.

Treatment of animals: In case of two weeks protocol, Swiss Albino rats were randomly assigned into 6 groups (A, B, C, D, E and F), six rats in each group for repeated dose treatment for two weeks to 
observe the effects of different doses of crude extract on blood glucose level, lipid profile and liver glycogen content in alloxan induced diabetic rats. The treatment groups were as follows: Group A: Normal; Group B: Diabetic control; Group C: Diabetic + Metformin (15 mg/kg BW); Group D: Diabetic + Bitter melon extract $(75 \mathrm{mg} / \mathrm{kg} \mathrm{BW})$; Group E: Diabetic + BME (150 mg/kg BW); Group F: Diabetic + BME (300 mg/kg BW).

In case of four weeks protocol, rats were randomly assigned into 5 groups (A, B, C, D and E), six rats in each group for repeated dose treatment for four weeks to determine the effect of therapy on blood glucose level, lipid profile and liver glycogen in alloxan induced diabetic rats. The treatment groups were as follows: Group A: Normal; Group B: Diabetic control; Group C: Diabetic + Metformin (15 $\mathrm{mg} / \mathrm{kg} \mathrm{BW})$; Group D: Diabetic + BME (300 mg/kg BW); Group E: Diabetic + Combination (Metformin $7.5 \mathrm{mg} / \mathrm{kg} \mathrm{BW}+$ BME $150 \mathrm{mg} / \mathrm{kg} \mathrm{BW}$ ).

At the end of the treatment period, the animals were fasted for at least 16 hours and sacrificed. Blood samples were collected in centrifuge tubes without anticoagulants and allowed to clot. The clotted blood was then centrifuged at $4000 \mathrm{rpm}$ for $30 \mathrm{~min}$. Serum was separated and then quickly stored at refrigerator for biochemical analysis.

Biochemical analysis: Serum glucose was determined by the glucose oxidase method using Clever Check glucose test meter (Bioland, Germany). Serum high density lipoprotein cholesterol (HDL-C) and low density lipoprotein cholesterol (LDL-C) level were estimated by the UV spectrophotometric method using HDL and LDL cholesterol liquicolor test kit (Human, Germany). Serum total cholesterol (TC) and triglycerides (TG) were estimated by the methods of UV spectrophotometric using diagnostic kits (Human, Germany).

Glycogen content in liver was measured according to Tarnoky et al., (1963) method which used spectrophotometric analysis to determine liver glycogen level using o-toluidine reagent. It utilizes the o-toluidine glucose coupling reactions for the estimation of glycogen after trichloroacetic acid extraction, precipitation by alcohol and hydrolysis.

Statistical analysis: The results are expressed as mean \pm SEM using Graph Pad Prism (version 4.0) computer program (Graph pad Software San Diego, CA, USA). We used a one-way analysis of variance (ANOVA), followed by Dunnett's post-hoc test or students paired or unpaired t-test where appropriate. The statistical method applied in each analysis was described in each figure. Results were considered to be significant when $p$ values were less than 0.05 $(\mathrm{p}<0.05)$.

\section{Results}

The effects of drug alone (metformin), bitter mellon extract (BME) and combination (metformin with BME) on the parameters of blood glucose level, lipid profile (TC, TG, LDL-C, HDL-C) and liver glycogen were performed for both short and longterm alloxan induced diabetes rats (AIDRs). AIDRs were treated with BME at different concentration (75, 150 and $300 \mathrm{mg} / \mathrm{kg} \mathrm{BW}$ ) for two weeks to determine the appropriate dose at which most significant results were obtained and the determined dose was used to carry out the research for next four weeks protocol.

\section{Two weeks treatment protocol}

Selection of dose of Momordica charantia: AIDRs were treated with methanolic extract of fresh fruits of $M$ charantia (bitter melon) at different doses $(75,150$ and $300 \mathrm{mg} / \mathrm{kg} \mathrm{BW})$ for two weeks on blood glucose level, lipid profile and liver glycogen level to determine the appropriate dose at which most significant results were obtained.

The effects of repeated dose treatment of BME for two weeks on blood glucose level in normal and alloxan induced diabetic rats (AIDRs) were shown in the Table 1. Intraperitoneal injection of alloxan in rats significantly increased the blood glucose level $(21.10 \pm 0.79 \mathrm{mmol} / \mathrm{L})$ in comparison to normal rats $(6.13 \pm 0.38 \mathrm{mmol} / \mathrm{L})$. Most significant decrease in blood glucose level had been observed for BME treated groups at dose $300 \mathrm{mg} / \mathrm{kg} \mathrm{BW}$ (from $22.40 \pm$ $0.44 \mathrm{mmol} / \mathrm{L}$ to $11.63 \pm 0.64 \mathrm{mmol} / \mathrm{L}$ ) in AIDRs. 
Induction of alloxan in rats for two weeks significantly altered lipid profile when compared with normal rats (Table 2). We studied the effects of different doses of BME (75, 150 and $300 \mathrm{mg} / \mathrm{kg} \mathrm{BW})$ on lipid profile (e.g. TC, TG, LDL-C and HDL-C levels) to select the most effective dose of BME in AIDRs. We observed that BME at different doses significantly changed the lipid profile and most significant change of lipid profile was found at 300 $\mathrm{mg} / \mathrm{kg} \mathrm{BW}$ among three doses. The BME $(300 \mathrm{mg} / \mathrm{kg}$ BW) reduced TC level 24.42\%, TG level $23.03 \%$, and LDL-C level $37.49 \%$, whereas the BME increased HDL-C level $43.25 \%$ when compared with AIDRs. As the effect of BME on lipid profile was dose dependent, we selected the most effective dose (300 mg/kg bw) for further study.

Table 1. Data for the blood glucose level (BGL) in normal and AIDRs after two weeks treatment.

\begin{tabular}{lcc}
\hline \multicolumn{1}{c}{ Treatment } & 0-Day & 14-Days \\
\hline Normal & $6.13 \pm 0.38$ & $6.17 \pm 0.20$ \\
AIDRs & $21.10 \pm 0.79^{\#}$ & $23.33 \pm 0.55^{*}$ \\
Alloxan + Metformin & $19.87 \pm 0.78$ & $12.67 \pm 0.58^{* *}$ \\
Alloxan + BME 75 & $18.07 \pm 0.81$ & $15.93 \pm 0.85^{*}$ \\
Alloxan + BME 150 & $21.63 \pm 1.24$ & $16.57 \pm 0.43^{*}$ \\
Alloxan + BME 300 & $22.40 \pm 0.44$ & $11.63 \pm 0.64^{* *}$ \\
\hline
\end{tabular}

The data were presented as mean $\pm \mathrm{SEM} ; \mathrm{n}=6$ in each group, ${ }^{*} \mathrm{p}<0.05$, $* * \mathrm{p}<0.01$ compared to diabetic control group (One way ANOVA followed by Dunnett's test). ${ }^{\#} \mathrm{p}<0.05$ vs normal group.

Table 2. Data for the lipid profile level in normal and AIDRs after two weeks treatment.

\begin{tabular}{|c|c|c|c|c|}
\hline \multirow[t]{2}{*}{ Treatment } & \multicolumn{2}{|c|}{$\mathrm{TC}$} & \multicolumn{2}{|c|}{$\mathrm{TG}$} \\
\hline & Mean \pm SEM & (\%) Inhibition & Mean \pm SEM & (\%) Inhibition \\
\hline Normal & $151.30 \pm 2.66$ & & $131.32 \pm 1.52$ & \\
\hline AIDRs & $219.19 \pm 2.53^{\#}$ & & $174.73 \pm 2.01^{\#}$ & \\
\hline Alloxan + Metformin & $162.09 \pm 3.59 *$ & 26.05 & $138.51 \pm 2.35^{*}$ & 20.73 \\
\hline Alloxan + BME 75 & $186.81 \pm 4.18$ & 14.77 & $147.99 \pm 1.75^{*}$ & 15.30 \\
\hline Alloxan + BME 150 & $171.01 \pm 3.53$ & 21.98 & $165.52 \pm 2.80$ & 5.27 \\
\hline Alloxan + BME 300 & $165.67 \pm 3.07 *$ & 24.42 & $134.48 \pm 1.79 *$ & 23.03 \\
\hline \multirow[t]{2}{*}{ Treatment } & \multicolumn{2}{|c|}{ LDL-C } & \multicolumn{2}{|c|}{ HDL-C } \\
\hline & Mean \pm SEM & (\%) Inhibition & Mean \pm SEM & $(\%)$ Increase \\
\hline Normal & $67.87 \pm 3.42$ & & $57.17 \pm 1.06$ & \\
\hline AIDRs & $155.01 \pm 2.59^{\#}$ & & $29.23 \pm 0.72^{\#}$ & \\
\hline Alloxan + Metformin & $89.99 \pm 3.30^{* *}$ & 41.94 & $44.39 \pm 1.06 * *$ & 51.84 \\
\hline Alloxan + BME 75 & $121.17 \pm 3.77$ & 21.83 & $36.04 \pm 2.47$ & 23.28 \\
\hline Alloxan + BME 150 & $105.84 \pm 2.94$ & 31.71 & $32.07 \pm 0.94$ & 9.69 \\
\hline Alloxan + BME 300 & $96.89 \pm 1.36^{* *}$ & 37.49 & $41.88 \pm 1.59 * *$ & 43.25 \\
\hline
\end{tabular}

The data were presented as mean $\pm \mathrm{SEM} ; \mathrm{n}=6$ in each group, ${ }^{*} \mathrm{p}<0.05,{ }^{*} \mathrm{p}<0.01$ compared to diabetic control group (One way ANOVA followed by Dunnett's test). ${ }^{\#} \mathrm{p}<0.05$ vs normal group. 


\section{Four weeks treatment protocol}

In our two weeks protocol, we observed the effects of different doses of bitter melon crude extract on AIDRs and found that BME dose at $300 \mathrm{mg} / \mathrm{kg}$ BW exhibited significant effectiveness and showed very close activities in comparison with metformin. That is why, we selected $300 \mathrm{mg} / \mathrm{kg}$ bw dose of BME for our four weeks protocol.

Effect on blood glucose level: The effects of repeated dose treatment of metform, BME and combination for four weeks on blood glucose level in normal and AIDRs are shown in the Table 3 . Intraperitoneal injection of alloxan in rats significantly increased the blood glucose level (18.85 $\pm 1.35 \mathrm{mmol} / \mathrm{L})$ in comparison to normal rats $(6.63 \pm$ $0.21 \mathrm{mmol} / \mathrm{L}$ ). Remarkable decrease in blood glucose level had been observed for metformin (10.2 \pm 0.42 $\mathrm{mmol} / \mathrm{L})$, BME (11.27 \pm 0.68$)$ and combination therapy treated groups $(7.65 \pm 0.23 \mathrm{mmol} / \mathrm{L})$ in
AIDRs. Our results suggested that BME when combined with metformin more effectively reduced the blood glucose level than mono-therapy in longterm alloxan-induced diabetic rats.

Table 3. Data for the blood glucose level (BGL) in normal and AIDRs after four weeks treatment.

\begin{tabular}{lcc}
\hline Treatment & 0-Day & 28-Days \\
\hline Normal & $6.63 \pm 0.21$ & $6.80 \pm 0.39$ \\
AIDRs & $18.85 \pm 1.35^{\#}$ & $18.42 \pm 0.95^{\#}$ \\
Alloxan + Metformin & $19.47 \pm 1.12$ & $10.20 \pm 0.42^{*}$ \\
Alloxan + BME 300 & $20.90 \pm 1.50$ & $11.27 \pm 0.68^{*}$ \\
Alloxan + Metformin & $16.45 \pm 0.31$ & $7.65 \pm 0.23^{* *}$ \\
+ BME & & \\
\hline
\end{tabular}

The data were presented as mean $\pm \mathrm{SEM} ; \mathrm{n}=6$ in each group, $* \mathrm{p}<0.05, * * \mathrm{p}<0.01$ compared to diabetic control group (One way ANOVA followed by Dunnett's test). \# $\mathrm{p}<0.05$ vs normal group.
A

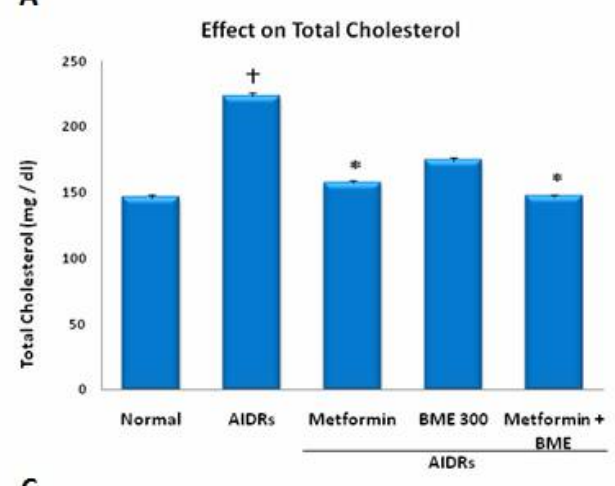

C

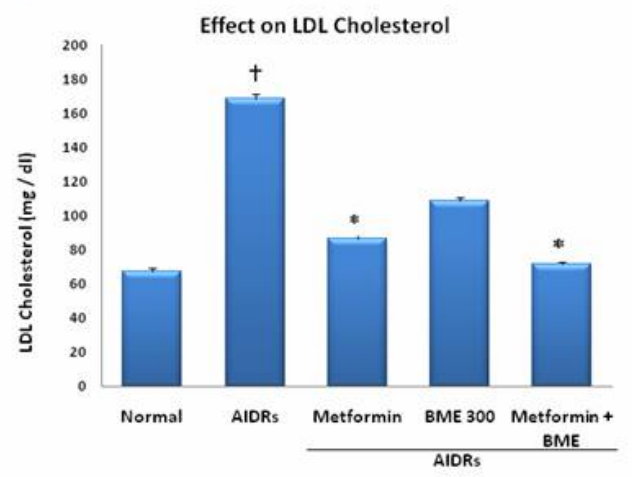

B
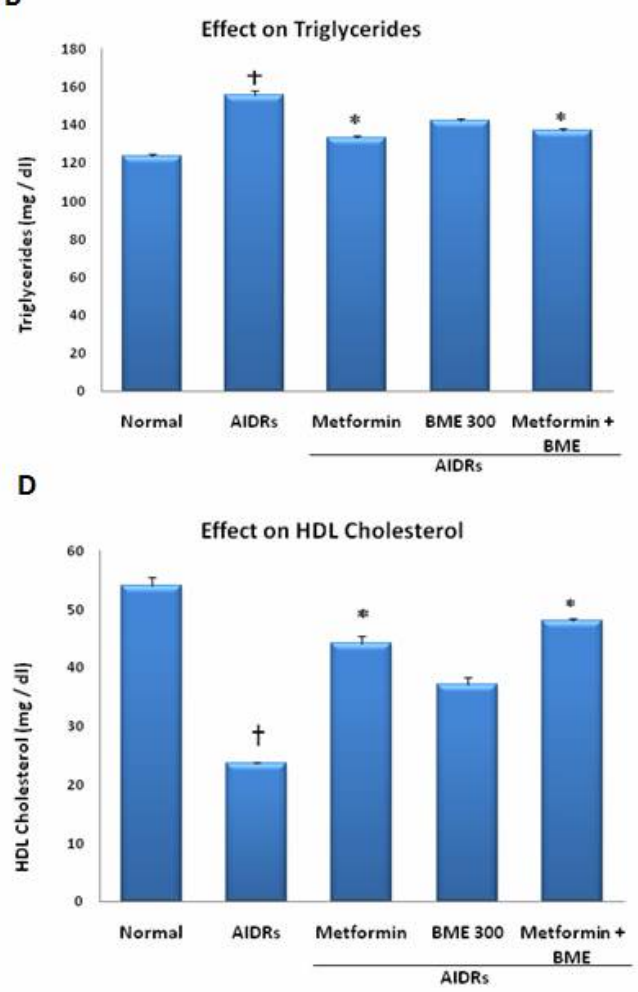

Figure 1. Effects of metform, BME and combination of both on lipid profile. A) TC, B) TG, C) LDL-C and D) HDL-C in AIDRs for four weeks. Data were presented as mean \pm SEM; $n=6$ in each group. ${ }^{*} \mathrm{p}<0.05$ and ${ }^{* *} \mathrm{p}<0.01$ compared to AIDRs. $\dagger(\mathrm{p}<0.05)$ compared to normal group. 
Effects on lipid profile: To investigate the effects of mono and combination therapy on lipid profile, we examined TC, TG, LDL-C and HDL-C level, after four weeks treatment with BME and metformin alone and combination of both in long-term AIDRs. It was found that BME, metformin and combination of both reduced TC level 29.57, 22.04 and 34.25 (Figure 1A), TG level 14.59, 8.70 and 11.92\% (Figure 1B), and LDL-C level 48.80, 35.71 and 57.73\% (Figure 1C), and they increased HDL-C level 46.58, 30.59 and $55.48 \%$ (Figure 1D), respectively when compared with diabetic rats. Here, the obtained results also suggested that the effect of combination therapy on lipid profile was higher than that of mono-therapy alone. These results showed that combination of
BME with metformin was more effective in lipid profile management than monotherapy alone in longterm AIDRs.

Effect on liver glycogen level: The effects of repeated dose treatment of metformin, BME and their combination for four weeks on liver glycogen level in normal and AIDRs are shown in the Figure 2. After four weeks treatment it was found that metformin and BME increased liver glycogen level $35.21 \%$ and $22.54 \%$, respectively, and their combination increased liver glycogen level $49.01 \%$. This result revealed that metformin when combined with BME showed better glycemic control than that of metform and BME alone in long-term AIDRs therapy.

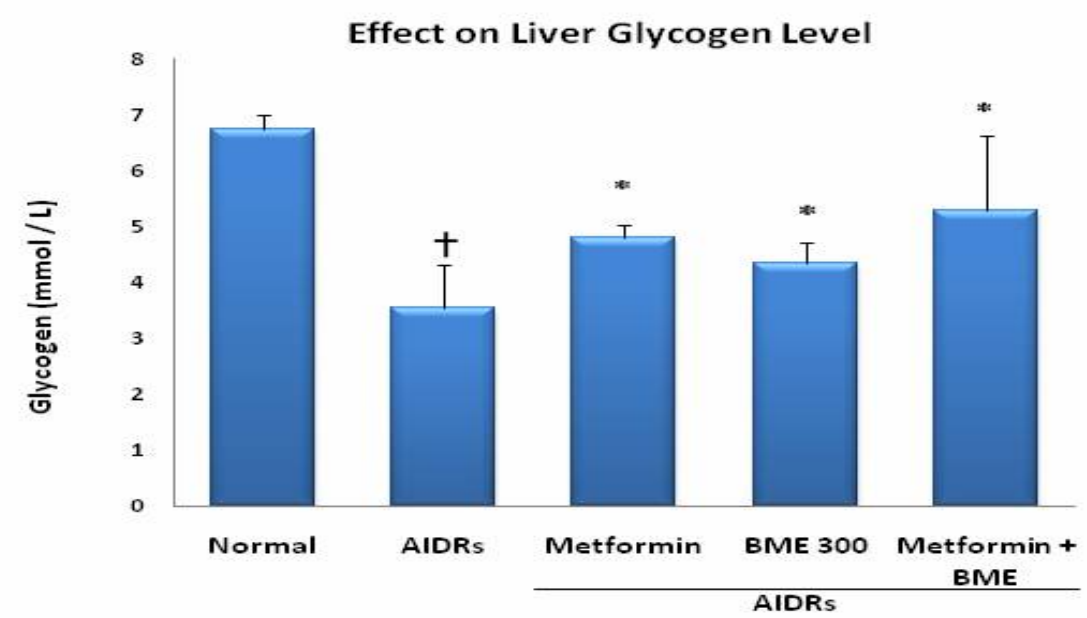

Figure 2. Effects of repeated dose treatment of metformin, BME and their combination for four weeks on liver glycogen level in AIDRs. The data were presented as mean \pm SEM; $n=6$ in each group, ${ }^{*} p<0.05$ compared to diabetic control group. $\dagger(\mathrm{p}<0.05)$ compared to normal group.

\section{Discussion}

Diabetes mellitus is a group of metabolic diseases characterized by hyperglycemia resulting from defects in insulin secretion, insulin action or both. The metabolic complications associated with obesity, hypertension, hyperlipidemia and the potential for atherosclerotic heart diseases are on the rise in the pediatric populations (Lee and Sanders, 2012). Diabetes mellitus is affecting approximately $8.3 \%$ of the world population (Hameed et al. 2015). Although insulin and other major classes of synthetic oral hypoglycaemic agents currently available for the management or control of both types of diabetes, however these pharmaceutical products are usually accompanied by some serious adverse effects. That is why, scientists and researchers are now focus on to develop different new drug combination therapy and natural plant products all over the world. The combination therapy (bitter melon crude extract and metformin) can be used as an effective medicine in the treatment of diabetes and diabetes related other complications such as liver toxicity and cardiovascular diseases. This research work has evaluated the antidiabetic and hypolipidemic effect of methanolic extract of $M$. charantia, metformin and their combination therapy. 
Alloxan monohydrate is one of the most frequently used inducer of experimental diabetes. It has been shown that the inhibition of mechanism of oxidative phosphorylation in the beta cells is the primary cause of diabetogenic action of alloxan (Lenzen, 2008). The present study showed that three different doses of BME produced significant dosedependent reduction in blood glucose level in AIDRs in comparison with metform. In addition, BME also showed dose-dependent antidyslipidemic effect in AIDRs. Our study revealed that BME $300 \mathrm{mg} / \mathrm{kg}$ BW was the most effective dose for glycemic control and improvement of lipid profile in AIDRs. BME $300 \mathrm{mg} / \mathrm{kg}$ BW dose had been selected by considering overall safety and efficacy for the long term treatment in our study.

Combination of BME with metformin produced a significant decrease in the blood glucose level which is higher than that produced by either drug alone. Thus it seems likely that, apart from pancreatic action, BME may also possess extra-pancreatic action, which could have contributed to its hypoglycemic action. Multiple mechanisms have been proposed as the cause of bitter melon's hypoglycemic properties. Electrophoresis and IR spectrum analysis have shown that components of bitter melon extract have structural similarities to animal insulin and have some insulin-like properties (Wong et al., 1985). Bitter melon may increase pancreatic insulin secretion and sensitivity, decrease hepatic gluconeogenesis, increase hepatic glycogen synthesis, and increase peripheral glucose oxidation in erythrocytes and adipocytes (Welihinda et al., 1982). Hypoglycemic activity of BME was suggested due to presence of polypeptide-p and a mixture of two steroid glycosides referred to as charantin in its extract (Khanna et al., 1981).

Diabetes is characterized by insulin deficiency which causes increased lipolysis in adipose issues and increased entry of free fatty acids (FFA) to the liver (Ohno et al., 2000). Increased FFA and insulin independent glucose uptake in the liver is reported to stimulate triglyceride synthesis (Coppack et al., 1984). This increased synthesis of triglycerides tends to reduce protein content of lipoproteins, especially in very low-density lipoprotein (VLDL) and LDL while the triglyceride content increases (Winocour et al., 1992). In this study, diabetic control rats showed significant changes in lipid abnormalities. It was found that combination therapy significantly reduced TC and LDL-cholesterol levels and increased HDL-cholesterol level but moderately decreased TG level than BME and metformin monotherapy in AIDRs compared to their diabetic control group. Hypolipidemic effects of $M$. charantia are reported to arise from enhanced oxidation of lipids associated with mitochondrial uncoupling and enhanced transport of lipid in liver and adipose tissue. Bitter melon contains a number of active ingredients like momordin, charantin and cucurbitane glycosides peptides that have antilipolytic and lipogenic activities (Chaturvedi and Georg, 2010).

Decreased glycolysis, impeded glycogenesis, and increased gluconeogenesis and glycogenolysis are some of the changes of glucose metabolism in the diabetic liver. In diabetics due to lack of availability of circulating insulin marked glycogenolysis is occurred to compensate the energy requirement of the body and hence liver glycogen level significantly reduced (Fernandes et al., 2007). Induction of diabetes with alloxan was associated with decrease in hepatic glycogen, which could be attributed to the decrease in the availability of the active form of enzyme glycogen synthetase probably because of low levels of insulin. In the present study, BME with metformin restored the depressed hepatic glycogen levels more effectively than the either drug alone possibly by increasing the level of insulin. Our results showed that supplementation of diabetic rats with combination therapy resulted in significant elevation in hepatic glycogen content.

Decreased in the activities of the enzymes involved in glucose homeostasis in liver and kidney such as hexokinase has been reported in diabetic animals resulting in depletion of liver and muscle glycogen content (Grover et al., 2000) Treatment with BME in combination with antidiabetic agents might increase the level of enzyme to the control 
level indicating an over-all increase in glucose influx. However, the exact mechanism of action needs further investigation.

\section{Conclusion}

Our present study was carried out to find out the combined beneficial effects of methanolic extract of $M$. charantia and metformin on glycemic control and improvement of lipid profile in diabetic rats. This study clearly indicated that the bitter melon crude extract possessed antidiabetic activity as well as antidyslipidemic effects in AIDRs. Again when it was administered in combination with metformin it showed safer, synergistic and promising hypoglycemic properties and reduced dose level of oral hypoglycemic agents, while giving better glycaemic control. It also maintained normal lipid profiles which may represent a protective mechanism against the development of atherosclerosis, and may effectively normalized the impaired liver glycogen level in alloxan induced diabetic rats. Hence, this combination therapy may be a cost effective, less toxic and wonderful remedy for the treatment of diabetes and hyperlipidemia, and bitter melon may be pursued for its clinical usefulness in the management of diabetes mellitus and other associated complications. A similar study in human subjects is desirable to determine if these results can be appropriately extrapolated to human diabetes. We need further study to determine the mechanism of action responsible for the anti-diabetic activity.

\section{Acknowledgement}

The authors thank Square Pharmaceuticals Ltd, Bangladesh for its kind gift of metformin.

\section{References}

Ali, Y., Alam, A.H.M.K., Rahman, M.A.A., Islam, M.S., Mamun, A., Khaled, M.A., Hossain, A.K.M.M. and Rashid, M. 2013. Inhibitory efffects of Nigella sativa seed extract on adrenaline-induced hyperlipidemia with cardiac hypertrophy in rats. J. Sci. Res. 5, 325334.
Carmena, R. 2005. Type 2 diabetes, dyslipidemia, and vascular risk: Rationale and evidence for correcting the lipid imbalance. Am. Heart J. 150, 859-870.

Chang, C.L., Lin, Y., Bartolome, A.P., Chen, Y.C., Chiu, S.C. and Yang, W.C. 2013. Herbal therapies for type 2 diabetes mellitus: chemistry, biology, and potential application of selected plants and compounds. Evid. Based Complement. Alternat. Med. 2013, 378657.

Chaturvedi, P. and Georg, S. 2010. Momordica charantia Maintains Normal Glucose Levels and Lipid Profiles and Prevents Oxidative Stress in Diabetic Rats Subjected to Chronic Sucrose Load. J. Med. Food. 13, 520-527.

Cho, N.H., Shaw, J.E., Karuranga, S., Huang, Y., da Rocha Fernandes, J.D., Ohlrogge, A.W. and Malanda, B. 2018. IDF Diabetes Atlas: Global estimates of diabetes prevalence for 2017 and projections for 2045 . Diabetes Res. Clin. Pract. 138, 271-281.

Coppack, S.W., Jensen, M.D. and Miles, J.M. 199.) In Vivo Regulation of Lipolysis in Humans. J. Lipid Res. 35, 177-193.

DeFronzo, R.A., Bonadonna, R.C. and Ferrannini, E. 1992. Pathogenesis of NIDDM: A balanced overview. Diabet. Care. 15, 318-368.

Fernandes, N.P.C., Lagishetty, C.V., Panda, V.S. and Naik, S.R. 2007. An experimental evaluation of the antidiabetic and antilipidemic properties of a standardized Momordica charantia fruit extract. BMC Complement. Alternat. Med. 7, 29.

Governa, P., Baini, G., Borgonetti, V., Cettolin, G., Giachetti, D., Magnano, A.R., Miraldiand, E. and Biagi, M. 2016. Phytotherapy in the Management of Diabetes: A Review. Molecules. 23, 105.

Grover, J.K., Vats, V. and Rathi, S.S. 2000. Antihyperglycemic effect of Eugenia jambolana and Tinosporacardifolia in experimental diabetes and their effects on key metabolic enzymes involved in carbohydrate metabolism. J. Ethnopharmacol. 73, 461-470.

Hameed, I., Masood,i S.R., Mir, S.A., Nabi, M., Ghazanfar, K. and Ganai, B.A. 2015. Type 2 diabetes mellitus: From a metabolic disorder to an inflammatory condition. W. J. Diabet. 6, 598-612.

Johansen, J.S., Harris, A.K., Rychly, D.J. and Ergul, A. 2005. Oxidative stress and the use of antioxidants in diabetes: linking basic science to clinical practice. Cardiovasc. Diabetol. 4, 5.

Khanna, P., Jain, S.C., Panagariya, A. and Dixit, V.P. 1981. Hypoglycemic activity of polypeptide-p from a plant source. J. Nat. Prod. 44, 648-55. 
Lamb, R.E. and Goldstein, B.J. 2008. Modulating an oxidative-inflammatory cascade: potential new treatment strategy for improving glucose metabolism, insulin resistance, and vascular function. Int. J. Clin. Pract. 62, 1087-1095.

Lee, L. and Sanders, R.A. 2012. Metabolic Syndrome, Pediatr. Rev. 33, 459 -468.

Lenzen, S. 2008. The mechanisms of alloxan-and streptozotocin-induced diabetes. Diabetol. 51, 216-26.

Ohno, T., Horio, F., Tanaka, S., Terada, M., Namikawa, T. and Kitoh, J. 2000. Fatty Liver and Hyperlipidemia in IDDM (Insulin-Dependent Diabetes Mellitus) of Streptozotocin-Treated Shrews. Life Sci. 66, 125-131.

Rahman, A.F.M.T., Islam, M.S., Ali, M.H., Alam, A.H.M.K., Rahman, M.A.A., Sadik, G. and Rashid, M. 2013. Nigella sativa oil potentiates the effects of pioglitazone on long term alloxan-induced diabetic rats. Bang. Pharm. J. 16, 143-151.

Sastri, B.N. 1962. Wealth of India-Raw Materials. Council of Scientific and Industrial Research, India; pp. 376.

Sekar, D.S., Sivagnanam, K. and Subramanian, S. 2005. Antidiabetic activity of $M$. charantia seeds on streptozotocin induced diabetic rats. Pharmazie. 60, 383-387.
Tarnoky, K. and Nagy, S. 1963. Spectrophotometric determination of glycogen with O-toludine. Clin. Chim. Acta. 8, 627-628.

Ullah, A., Khan, A. and Khan, S. 2016. Diabetes mellitus and oxidative stress - a concise review. Saudi Pharm. J. 24, 547-553.

Welihinda, J., Arvidson, G. and Gylfe, E., Hellman, B. and Karlsson, E. 1982. The insulin-releasing activity of the tropical plant Momordica charantia. Acta. Biol. Med. Ger. 41, 1229-40.

WHO and IDF, 2004. Diabetes action now: An initiative of the World Health Organization and the International Diabetic Federation. pp. 1-20.

Winocour, P.H., Durrington, P.N., Bhatnagar, D., Ishola, M., Arro, S.l. and Mackness, M. 1992. Abnormalities of VLDL, IDL, and LDL characterize insulindependent diabetes mellitus. Arteriosc. Thromb. 12, 920-928.

Wong, C.M., Yeung, H.W. and Ng, T.B. 1985. Screening of Trichosanthes kirilowii, Momordica charantia and Cucurbita maxima (family Cucurbitaceae) for compounds with antilipolytic activity. $J$. Ethnopharmacol. 13, 313-21. 